\title{
Early versus late awake prone positioning in non-intubated patients with COVID-19
}

\author{
Ramandeep Kaur ${ }^{1}$, David L. Vines', Sara Mirza², Ahmad Elshafei ${ }^{1}$, Julie A. Jackson³, Lauren J. Harnois', \\ Tyler Weiss' ${ }^{1}$, J. Brady Scott ${ }^{1}$, Matthew W. Trump ${ }^{4}$, Idrees Mogri ${ }^{5}$, Flor Cerda ${ }^{6}$, Amnah A. Alolaiwat ${ }^{1}$, \\ Amanda R. Miller ${ }^{1}$, Andrew M. Klein ${ }^{1}$, Trevor W. Oetting ${ }^{3}$, Lindsey Morris ${ }^{5}$, Scott Heckart ${ }^{3}$, Lindsay Capouch³, \\ Hangyong $\mathrm{He}^{7}$ and $\mathrm{Jie}_{\mathrm{Li}}{ }^{1^{*}}$ (B)
}

\begin{abstract}
Background: Awake prone positioning (APP) is widely used in the management of patients with coronavirus disease (COVID-19). The primary objective of this study was to compare the outcome of COVID-19 patients who received early versus late APP.

Methods: Post hoc analysis of data collected for a randomized controlled trial (ClinicalTrials.gov NCT04325906). Adult patients with acute hypoxemic respiratory failure secondary to COVID-19 who received APP for at least one hour were included. Early prone positioning was defined as APP initiated within $24 \mathrm{~h}$ of high-flow nasal cannula (HFNC) start.

Primary outcomes were 28-day mortality and intubation rate.

Results: We included 125 patients (79 male) with a mean age of 62 years. Of them, 92 (73.6\%) received early APP and 33 (26.4\%) received late APP. Median time from HFNC initiation to APP was $2.25(0.8-12.82)$ vs 36.35 (30.2-75.23) hours in the early and late APP group ( $p<0.0001)$, respectively. Average APP duration was 5.07 (2.0-9.05) and 3.0 (1.09-5.64) hours per day in early and late APP group $(p<0.0001)$, respectively. The early APP group had lower mortality compared to the late APP group ( $26 \%$ vs $45 \%, p=0.039$ ), but no difference was found in intubation rate. Advanced age (OR 1.12 [95\% Cl 1.0-1.95], $p=0.001$ ), intubation (OR 10.65 [95\% Cl 2.77-40.91], $p=0.001$ ), longer time to initiate APP (OR 1.02 [95\% Cl 1.0-1.04], $p=0.047$ ) and hydrocortisone use (OR 6.2 [95\% Cl 1.23-31.1], $p=0.027$ ) were associated with increased mortality.
\end{abstract}

Conclusions: Early initiation ( $<24 \mathrm{~h}$ of HFNC use) of APP in acute hypoxemic respiratory failure secondary to COVID19 improves 28-day survival.

Trial registration ClinicalTrials.gov NCT04325906.

Keywords: Awake prone positioning, Non-intubated, COVID-19, Coronavirus, Acute hypoxemic respiratory failure

*Correspondence: Jie_Li@rush.edu

${ }^{1}$ Division of Respiratory Care, Department of Cardiopulmonary Sciences, Rush University Medical Center, 600 S Paulina St, Suite 765, Chicago, IL, USA

Full list of author information is available at the end of the article

\section{Introduction}

Coronavirus disease (COVID-19) is a viral infectious disease caused by coronavirus (SARS-CoV-2) [1]. COVID19 primarily affects the respiratory system causing mild to severe respiratory illness. Around $25-30 \%$ of COVID19 patients develop signs of acute respiratory distress requiring higher respiratory support in terms of oxygen therapy, noninvasive and invasive positive pressure ventilation [2]. Prone positioning improves oxygenation by the original author(s) and the source, provide a link to the Creative Commons licence, and indicate if changes were made. The images or other third party material in this article are included in the article's Creative Commons licence, unless indicated otherwise in a credit line to the material. If material is not included in the article's Creative Commons licence and your intended use is not permitted by statutory regulation or exceeds the permitted use, you will need to obtain permission directly from the copyright holder. To view a copy of this licence, visit http://creativecommons.org/licenses/by/4.0/. The Creative Commons Public Domain Dedication waiver (http://creativeco mmons.org/publicdomain/zero/1.0/) applies to the data made available in this article, unless otherwise stated in a credit line to the data. 
uniform distribution of tidal volume and recruitment of the dorsal lung regions leading to improved lung compliance [3]. Before the COVID-19 pandemic, a small, prospective observational study demonstrated the benefit of using prone positioning among non-intubated patients with moderate acute respiratory distress syndrome (ARDS) to reduce the need for invasive mechanical ventilation [4]. Since the emergence of COVID-19, this technique has been extensively used to improve oxygenation in non-intubated COVID-19 patients with acute hypoxemic respiratory failure (AHRF) [5].

There is evidence demonstrating the benefits of early prone positioning to improve oxygenation and patient outcomes in intubated patients with moderate to severe ARDS [6]. A recent multicenter cohort study investigating the timing of prone positioning initiation among mechanically ventilated patients with COVID-19 found a lower hospital mortality among those who received early prone positioning (within 2 days of ICU admission) [7]. Another retrospective, multicenter observational study included 827 non-intubated patients with COVID-19 and found that awake prone positioning (APP) was significantly associated with lower mortality $(20.0 \%$ vs $37.9 \% ; p<0.0001)$ and intubation rate $(23.6 \%$ vs $40.4 \%$; $p<0.0001)$ as compared to supine position [8]. Randomized controlled trials have been done to evaluate the feasibility of implementation and patient compliance with APP in patients with COVID-19, but no long-term outcomes were assessed [9-13]. A recent systematic review found that APP improved oxygenation among patients with AHRF due to COVID-19, however, APP did not reduce intubation rates [14]. Finally, a collaborative meta-trial of six randomized controlled superiority trials, on which this post hoc analysis is based, enrolled a total of 1121 patients and found hazard ratios of 0.75 (95\% CI, 0.62-0.91) for intubation and 0.87 (95\% CI, 0.68-1.11) for 28-day mortality with APP, as compared to the standard care group [15].

Despite multiple studies showing benefit of prone positioning among non-intubated patients with COVID-19, there is no clear evidence available guiding the timing of awake prone positioning for patients with COVID-19 to achieve optimal patient outcomes [16]. Therefore, the primary objective of this study was to compare early versus late initiation of awake prone positioning (APP) on patient outcomes, including hospital mortality and the need for invasive mechanical ventilation (IMV).

\section{Methods}

From April 2nd, 2020 to January 26th, 2021, we participated in a collaborative meta-trial of six randomized controlled open-label superiority trials [17] to compare the effectiveness of APP versus standard care in patients with AHRF due to COVID-19 supported with high-flow nasal cannula (HFNC). The intent of the unique study design was to achieve results with a sufficient effect size, in a faster manner, and at a lower cost [18]. It was felt this novel approach was necessary during a global event, like the COVID-19 pandemic. Four hospitals joined in the American trial (NCT04325906) and the randomization was assigned by the leading institution (Rush University Medical Center). The study protocol was approved by the institutional review board (20032604-IRB01). This post hoc analysis was conducted using the American data set.

\section{Study procedure}

In the American trial, patients were enrolled to receive APP or standard care if they were diagnosed with AHRF secondary to COVID-19 and had the ratio of saturation of pulse oximetry $\left(\mathrm{SpO}_{2}\right)$ to the fraction of inspired oxygen $\left(\mathrm{FiO}_{2}\right)<240$. All patients received respiratory support via high-flow nasal cannula (HFNC) initiated at 50 $\mathrm{L} /$ min with $\mathrm{FiO}_{2}$ titrated to maintain $\mathrm{SpO}_{2}$ between 90 and $95 \%$. HFNC was discontinued when the weaning criteria of $\mathrm{F}_{\mathrm{i}} \mathrm{O}_{2}$ at 0.4 and flow at $40 \mathrm{~L} / \mathrm{min}$ were met. For the patients who were randomized to the APP group, prone positioning was performed under clinician supervision and patients were instructed to maintain prone positioning as long as tolerated. In the standard care group, prone positioning was discouraged and if occurred, it was recorded as a protocol violation.

In this post hoc analysis, patients who received APP for a minimum of one hour were included, regardless of the group (APP or standard care) they were originally assigned. The subjects were excluded if the information on APP was missing.

\section{Data collection}

For the original study, demographic and clinical data were attained from the patient's electronic medical record. Demographic data included age, gender, ethnicity, height, and weight. Clinical data included medical history, medications list, laboratory, and microbiology findings. Data related to APP included vital signs and HFNC settings before and after the first APP session and start/end time for each prone session in the first three days of APP. ROX index was calculated using $\mathrm{SpO}_{2} / \mathrm{FiO}_{2}$ divided by respiratory rate [19]. The types of respiratory interventions including noninvasive and invasive positive pressure ventilation, use of inhaled vasodilator via HFNC or invasive ventilation, and the need for extracorporeal membrane oxygenation (ECMO) during hospitalization were recorded. Length of intensive care unit (ICU) and hospital stay, as well as the hospital outcome, were obtained. 


\section{Definitions and study outcome}

Early APP was defined as APP initiated within $24 \mathrm{~h}$ of starting HFNC therapy. The primary study outcomes were 28-day mortality and intubation rate among patients that received early vs late APP.

\section{Statistical analysis}

Continuous variables are presented as means \pm standard deviation (SD or as medians and interquartile ranges (IQR). Comparison of continuous variables between early vs late APP groups was conducted using Student's t test for variables with a normal distribution and using the Mann-Whitney U test for variables with a non-normal distribution. Results with respect to categorical variables are presented as proportions and were analyzed with chi-square or Fisher's exact tests. A multivariate logistic regression model was used to identify the risk factors for hospital death. Outcome variables and covariates were assessed using the enter method, model fit was assessed by Hosmer-Lemeshow goodness-of-fit statistic and model performance by the classification tables. Statistically significant independent variables were maintained in the model. Kaplan-Meier method was used to perform the survival analysis between the two study groups. All reported $\mathrm{p}$ values are two sided and a $p$ value $<0.05$ was considered significant. Statistical analysis was done using SPSS 26.0 for Windows (SPSS, Inc., Chicago, IL, USA).

\section{Results}

Subject characteristics

Between April 2nd, 2020 and January 26th, 2021, 222 patients with COVID-19 were enrolled in the American trial, 112 patients were assigned to receive APP and 110 patients were assigned to standard care, of whom 26 patients had protocol violation to receive rescue APP. Thus, 138 patients (112 from APP group and 26 from control group) were included for the post hoc analysis (Fig. 1). Of these 138 patients, 13 were excluded due to being self-proned for less than one hour $(n=10)$ and missing data $(n=3)$. A total of 125 patients were enrolled in this study, of whom 92 (73.6\%) received early APP and $33(26.4 \%)$ received late APP. Overall, the mean age of those included in this study was $62( \pm 11.9)$ years, 79 (63.2\%) patients were males and 70 (56\%) were of Hispanic/Latino ethnicity. The median sequential organ failure assessment (SOFA) score was 3 (IQR 2-4.5) at study enrollment for all patients included in this study. The median HFNC set flow was 50 (IQR 50-60) L/min and $\mathrm{FiO}_{2}$ was 0.6 (IQR 0.5-0.75) at study enrollment. Demographic and baseline clinical characteristics were similar in both groups (Table 1), except for fewer Hispanic patients $(33.3 \%$ vs $64.1 \%, p=0.002)$ and more Caucasian patients $(45.5 \%$ vs $23.9 \%, p=0.027)$ in the late prone group.

138 adult patients with COVID-19 received proning in awake proning trial

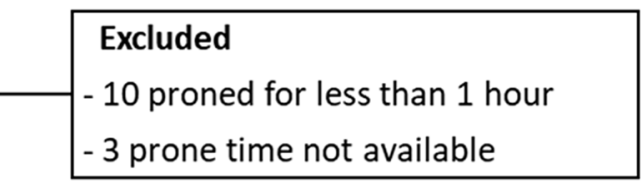

125 enrolled in the study

Early Awake Prone Positioning ( $n=92$ )

Fig. 1 Study flow diagram 
Time from HFNC initiation to APP

Overall, 67 (54\%) patients received APP within $11 \mathrm{~h}$ of HFNC initiation, 25 (20\%) within 12-23 h, 16 (13\%) within $24-35 \mathrm{~h}$ and 17 (14\%) after $36 \mathrm{~h}$ of initiating HFNC. The median time to start APP after initiating HFNC was $2.25 \mathrm{~h}$ in early APP group and $36.35 \mathrm{~h}$ among those who received late APP $(p<0.001)$ (Table 1$)$. The median time to start APP from hospital admission was $18 \mathrm{~h}$ in the early and $60 \mathrm{~h}$ in the late APP group $(p<0.001)$. The early APP group spent a median of $5.07 \mathrm{~h} /$ day and the late group spent a median of $3 \mathrm{~h} /$ day in the prone position. $(p=0.006)$.

\section{Oxygenation response}

There was no significant difference in the $\mathrm{SpO}_{2} / \mathrm{FiO}_{2}$ ratio or ROX index before APP in the early vs late APP groups;

Table 1 Overall subject baseline characteristics and comparison between the early and late awake prone positioning group

\begin{tabular}{|c|c|c|c|c|}
\hline Variables & $\begin{array}{l}\text { Overall } \\
(n=125)\end{array}$ & $\begin{array}{l}\text { Early awake prone } \\
(n=92)\end{array}$ & $\begin{array}{l}\text { Late awake prone } \\
(n=33)\end{array}$ & $P$ value \\
\hline Age, mean (SD) & $62.0 \pm 11.9$ & $61.1 \pm 12.3$ & $64.9 \pm 10.4$ & 0.113 \\
\hline Male, $n(\%)$ & $79(63.2)$ & $56(61)$ & $23(67)$ & 0.367 \\
\hline $\mathrm{BMI}\left(\mathrm{kg} / \mathrm{m}^{2}\right)$, mean (SD) & $30 \pm 5.0$ & $30.23 \pm 4.96$ & $29.47 \pm 5.14$ & 0.411 \\
\hline \multicolumn{5}{|l|}{ Ethnicity, $n(\%)$} \\
\hline Hispanic/Latino & $70(56)$ & $59(64.1)$ & $11(33.3)$ & 0.002 \\
\hline Caucasian & $37(29.6)$ & $22(23.9)$ & $15(45.5)$ & 0.027 \\
\hline African American & $7(5.6)$ & $5(5.4)$ & $2(6.1)$ & 0.59 \\
\hline Asian & $4(3.2)$ & $2(2.2)$ & $2(6.1)$ & 0.28 \\
\hline Unknown & $3(2.4)$ & $1(1.1)$ & $2(6.1)$ & 0.17 \\
\hline Others & $4(3.2)$ & $3(3.3)$ & $1(3)$ & 0.60 \\
\hline \multicolumn{5}{|l|}{ Comorbidities, n (\%) } \\
\hline Diabetes Mellitus & $54(43.2)$ & $17(51.5)$ & $37(40.2)$ & 0.261 \\
\hline Chronic Lung Disease & $17(13.6)$ & $10(11)$ & $7(21)$ & 0.119 \\
\hline Cardiovascular Disease & $29(23.2)$ & 18 (19.6) & $11(33.3)$ & 0.108 \\
\hline Chronic Renal Disease & $11(8.8)$ & $10(10.9)$ & $1(3)$ & 0.157 \\
\hline Chronic Liver Disease & $1(0.8)$ & $1(1.1)$ & 0 & 0.736 \\
\hline Immunocompromised Condition & $16(12.8)$ & $11(12)$ & $5(15)$ & 0.637 \\
\hline Neurologic disease & $5(4)$ & $4(4.3)$ & $1(3)$ & 0.603 \\
\hline Others & $25(20)$ & $23(25)$ & $2(6.1)$ & 0.020 \\
\hline Smoking status, $n(\%)$ & & & & 0.12 \\
\hline Current Smoker & $4(3.2)$ & $4(4.3)$ & 0 & \\
\hline Former Smoker & $38(30.4)$ & $23(25)$ & $15(45.5)$ & \\
\hline Never & $73(58.4)$ & $57(62)$ & $16(48.5)$ & \\
\hline Not available & $10(8)$ & $8(8.7)$ & $2(6)$ & \\
\hline SOFA score on admission, median (IQR) & $3(2-4.5)$ & $3(2-4.75)$ & $3(3-4.5)$ & 0.70 \\
\hline Assigned to APP group, $n(\%)$ & $101(80.8)$ & $88(96)$ & $13(39)$ & \\
\hline $\mathrm{SpO}_{2} / \mathrm{FiO}_{2}$ ratio on enrollment, median (IQR) & $143.8(117.5-174.4)$ & $135(116.2-166.5)$ & $155(131.6-188.5)$ & 0.052 \\
\hline Time from hospital admission to APP start (h), median (IQR) & $27.48(13.1-64.2)$ & $18(7.1-43.2)$ & $60(34.9-105)$ & $<0.001$ \\
\hline Time from HFNC start to APP (h), median (IQR) & $8.58(1.31-24.87)$ & $2.25(0.8-12.82)$ & $36.35(30.2-75.23)$ & $<0.001$ \\
\hline Total APP hours in the first three days, median (IQR) & $13.08(3.5-43.25)$ & $16(5.4-51.5)$ & $5(2.5-17.5)$ & 0.004 \\
\hline APP hours/day, median (IQR) & $4.45(1.75-8.37)$ & $5.07(2-9.05)$ & $3(1.09-5.64)$ & 0.006 \\
\hline HFNC duration (d), median (IQR) & $6(2.97-9.46)$ & $5(2.2-9)$ & $6(3.2-10.5)$ & 0.18 \\
\hline Antiviral therapy, $n(\%)$ & $84(67.2)$ & $65(70.7)$ & $19(57.6)$ & 0.12 \\
\hline Steroids use, $n(\%)$ & $93(74.4)$ & $64(69.6)$ & $29(87.9)$ & 0.039 \\
\hline Time from HFNC start to steroid start (h), median (IQR) & $-12.48(-25.3$ to 4.58$)$ & $-14.47(-33$ to 0$)$ & $-8.57(-20.8$ to 7.93$)$ & 0.19 \\
\hline \multicolumn{5}{|l|}{ Steroids type, $n(\%)$} \\
\hline Dexamethasone & $82(65.6)$ & $56(60.9)$ & $26(78.8)$ & 0.063 \\
\hline Hydrocortisone & $18(14.4)$ & $13(14.1)$ & $5(15.1)$ & 0.54 \\
\hline Methylprednisone/Prednisone & $15(12)$ & $10(10.9)$ & $5(15.1)$ & 0.35 \\
\hline
\end{tabular}


however, after $30 \mathrm{~min}$ in the first prone session, the early APP group had a higher $\mathrm{SpO}_{2} / \mathrm{FiO}_{2}$ ratio [163.2 (132.8211) vs 141.4 (105-172.5); $p=0.007$ ] (Fig. 2a) and ROX index [7.24 (5-9.93) vs 5 (3.8-6.95); $p=0.002$ ] (Fig. 3a). There was no significant difference in $\mathrm{SpO}_{2} / \mathrm{FiO}_{2}$ ratio change [15.22 (3.94-44) vs $6.83(2.1-19.24) ; p=0.076$ ] (Fig. 2b) between the two groups but the ROX index change was lower in the late APP group [(1.26 (0.312.89 ) vs 0.21 ( -1.57 to 1.1$) ; p=0.01$ ] (Fig. 3b).

\section{Study outcome}

As the duration between HFNC initiation to APP increased, the hospital mortality increased (Fig. 4a) with no impact on the intubation rate (Fig. 4b). The late APP group had a significantly higher mortality as compared to those who received early APP ( $45 \%$ vs $26 \%, p=0.039$; Table 2). The Kaplan-Meier survival plot demonstrated a decrease in survival among patients who received late APP (Fig. 5). The hospital and ICU length of stay were similar for the two groups. In terms of intubation rate and IMV duration, 48 (38.4\%) patients were intubated overall and there was no significant difference in intubation rate and IMV duration among the two study groups. The average time from HFNC initiation to intubation was similar in both groups with median time to intubation of $5.13(1.89-10.85)$ days in the early APP group and 5.27 (3.2-9.56) days in the late APP group $(p=0.65)$. Similarly,

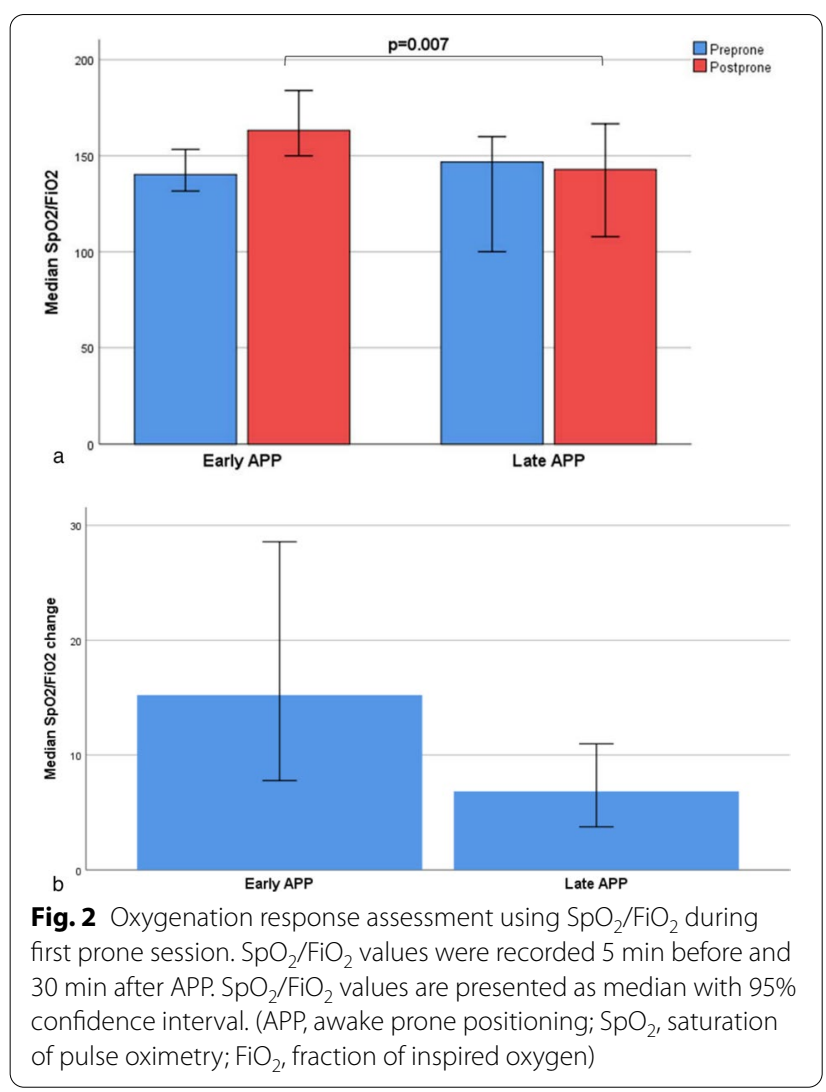

there was no difference in the two groups in terms of time from APP start to intubation. NIV use and duration from HFNC initiation to NIV were also similar among the two study groups. The use of rescue respiratory support such as ECMO and inhaled vasodilators was similar. There was no significant difference among the study groups in terms of antiviral therapy (remdesivir), however, more patients received steroids in the late APP group ( $87.9 \%$ vs $66.3 \%$, $p=0.039$ ).

\section{Risk factors associated with hospital death}

In the multivariate logistic regression analysis, factors independently associated with hospital mortality were advanced age (OR 1.12 [95\% CI 1-1.95], $p=0.001$ ), use of invasive mechanical ventilation (OR 10.65 [95\% CI 2.77-40.91], $p=0.001)$, longer time to initiate prone from HFNC start (OR 1.02 [95\% CI 1-1.04], $p=0.047$ ) and hydrocortisone use (OR 6.2 [95\% CI 1.23-31.1], $p=0.027)$.

\section{Discussion}

To our knowledge, this is the first study to explore the association between the time to initiate APP (early vs late) and mortality among non-intubated patients with COVID-19. In this post hoc analysis, we found that late APP was independently associated with an increase in hospital mortality, however, the association between

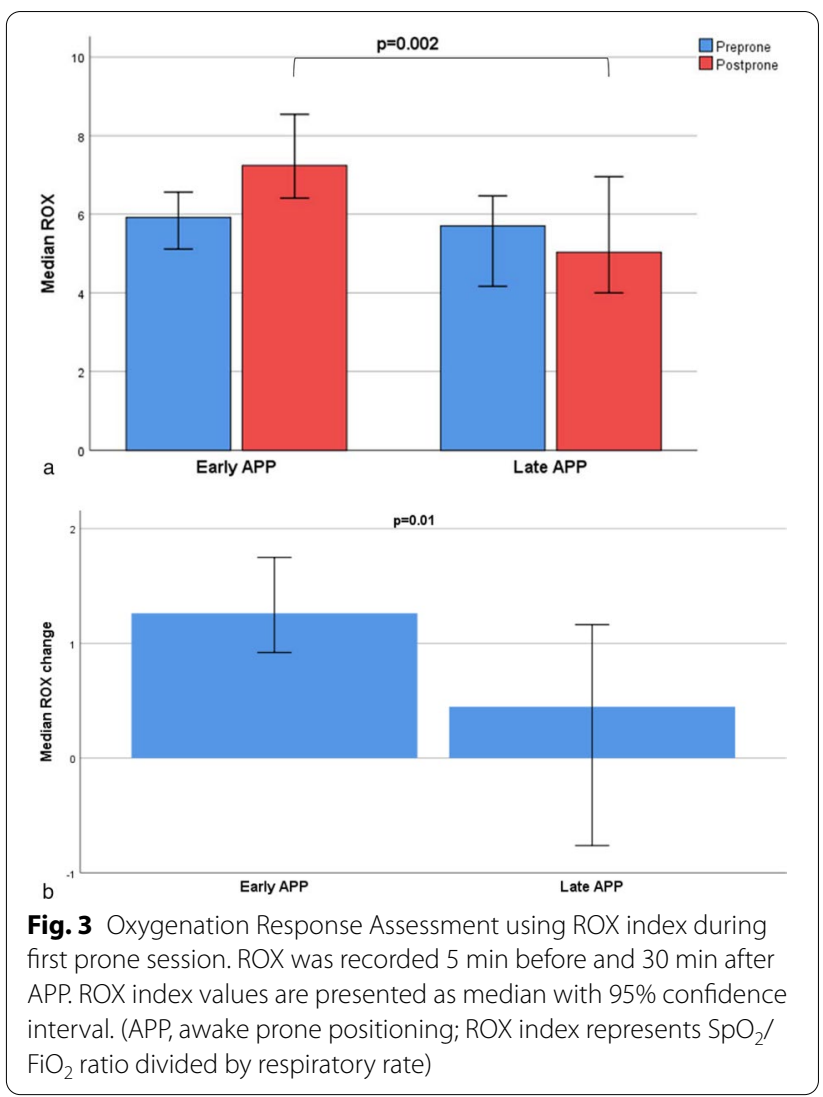




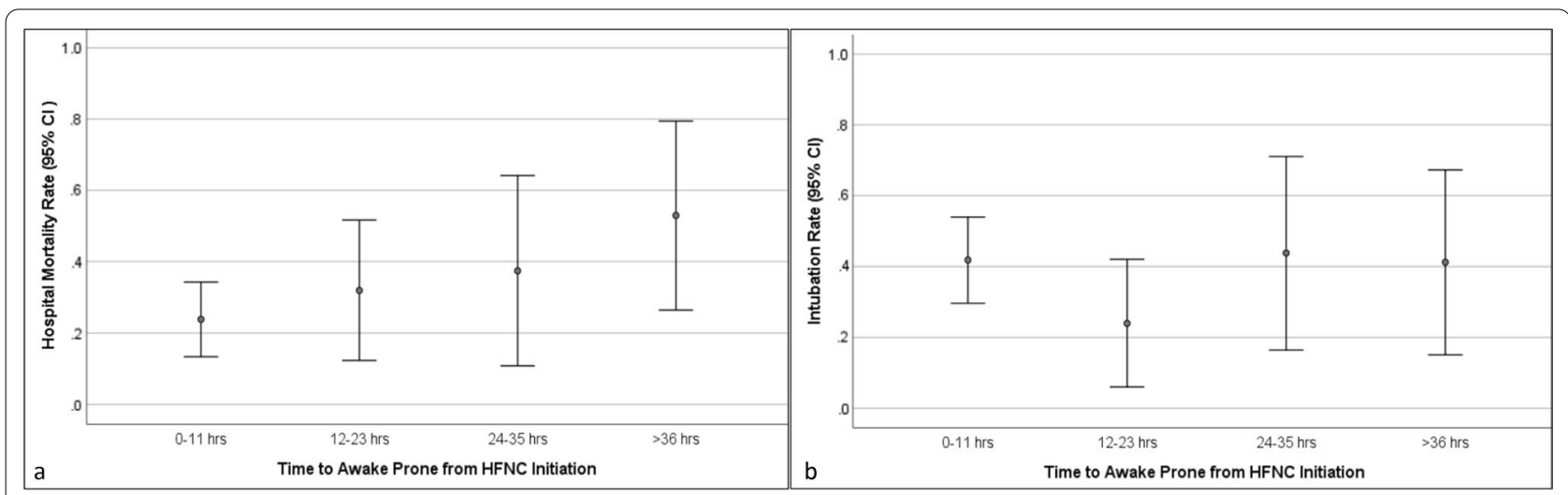

Fig. 4 Time to initiation of awake prone positioning and patient outcome (HFNC, high-flow nasal cannula; $\mathrm{Cl}$, confidence interval)

Table 2 Study outcome comparison between the early and late awake prone group

\begin{tabular}{llll}
\hline Outcomes & Early Awake Prone $(\boldsymbol{n}=\mathbf{9 2})$ & Late Awake Prone $(\boldsymbol{n}=\mathbf{3 3})$ & $\boldsymbol{P}$ value \\
\hline 28 day mortality, $n(\%)$ & $24(26)$ & $15(45)$ & 0.039 \\
Death without intubation, $n(\%)$ & $7(7.6)$ & $6(18.2)$ & 0.088 \\
Hospital LOS (d), median (IQR) & $13.97(9.64-24.9)$ & $12.53(9-20.9)$ & 0.66 \\
ICU LOS (d), median (IQR) & $7.91(4.25-21)$ & $14(4.38-16.9)$ & 0.55 \\
IMV use, $n$ (\%) & $34(37)$ & $8.89 \pm 6.10$ & 0.58 \\
IMV duration (d), median (IQR) & $10.59 \pm 6.12$ & $5.27(3.2-9.56)$ & 0.43 \\
Time from HFNC start to intubation (d), median (IQR) & $5.13(1.89-10.85)$ & $3.12(1.31-8.23)$ & 0.65 \\
Time from APP start to intubation (d), median (IQR) & $4.73(1.85-10.6)$ & $5(15.2)$ & 0.37 \\
NIV use, $n$ (\%) & $23(25)$ & $2.11(0.7-8.95)$ & 0.24 \\
Time from HFNC start to NIV (d), median (IQR) & $3.74(0.83-9.61)$ & 0 & 0.77 \\
ECMO use, $n$ (\%) & $2(2.2)$ & $9(27.3)$ & 0.54 \\
Inhaled vasodilator use, $n(\%)$ & $26(28.3)$ & 0.28 \\
\hline
\end{tabular}

LOS length of stay, IQR interquartile range, ICU intensive care unit, IMV invasive mechanical ventilation, NIV noninvasive ventilation, HFNC high-flow nasal cannula, ECMO extracorporeal membrane oxygenation, APP Awake Prone Positioning

exposure and outcome is weak (OR 1.02 [95\% CI $1-1.04])$. Patients who were proned within $24 \mathrm{~h}$ of initiating HFNC for AHRF (early APP) had a lower mortality than those who received APP after $24 \mathrm{~h}$ (late APP). Additionally, being elderly, intubated, and receiving hydrocortisone were significantly associated with mortality at 28 days.

Awake prone positioning is a noninvasive technique widely used among patients with COVID-19 and was found to reduce the need for invasive mechanical ventilation and improve patient outcomes in the recent large, meta-trial of six randomized controlled open-label superiority trials conducted across six countries [15]. The present study results suggest that initiating early prone positioning within $24 \mathrm{~h}$ of HFNC start further improves the 28-day mortality.

Several reasons may explain our research findings. First, COVID-19-induced lung injury is described to have two distinctive phenotypes, type $\mathrm{L}$ and type $\mathrm{H}[20]$.
Type L represents high compliance/low elastance, and low alveolar recruitability, while type $\mathrm{H}$ represents low compliance/high elastance and high alveolar recruitability [21]. Type $\mathrm{H}$ reportedly responds well to positive end expiratory pressure and prone positioning. This phenotype likely represents a time-related disease spectrum where the early use of HFNC and APP may improve ventilation-perfusion matching and reduce dyspnea related work of breathing and patient self-inflicted lung injury $[22,23]$. High respiratory drive, commonly found among patients with COVID-19, is associated with increased transpulmonary pressure changes that lead to vascular leakage and self-inflicted lung injury in patients with existing lung injury [24]. Furthermore, respiratory rates exceeding 22 breaths/minutes are associated with a 1.9-3.2 fold increase in mortality risk in patients with COVID-19 [25]. In the present study, we found that early use of APP led to significant improvements in the oxygenation status and work of breathing, assessed by $\mathrm{SpO}_{2} /$ 


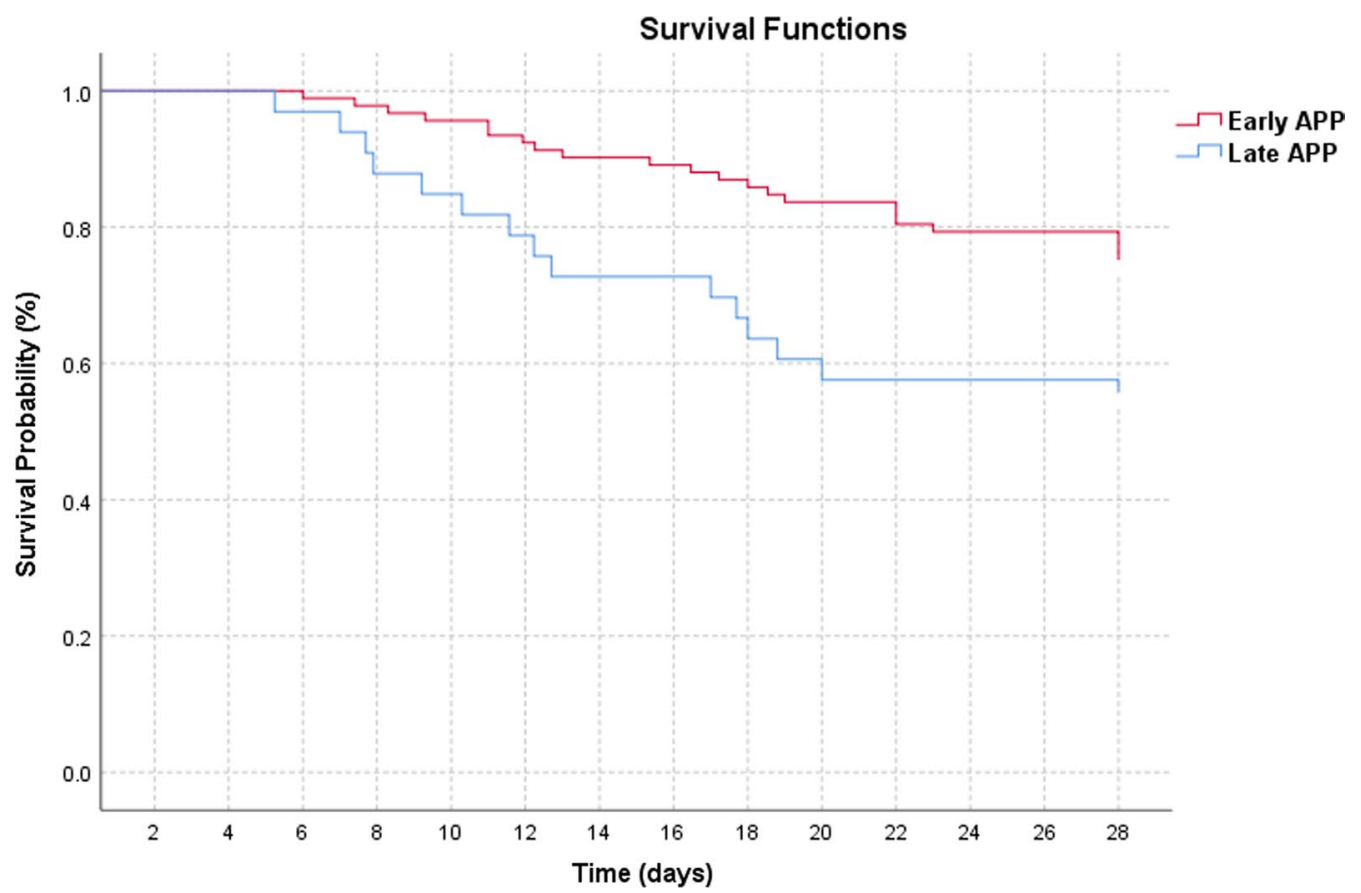

Fig. 5 Kaplan-Meier Survival probabilities over 28 days after hospital admission (APP, awake prone positioning)

$\mathrm{FiO}_{2}$ ratio and $\mathrm{ROX}$ index. Patients who received early APP had significantly higher $\mathrm{SpO}_{2} / \mathrm{FiO}_{2}$ ratios after being proned for $30 \mathrm{~min}$ as compared to those who received late prone positioning. Similarly, the ROX index, a predictor of outcome with the use of HFNC among patients with acute respiratory failure [19], was higher among patients that received early APP.

Based on morphology, early ARDS represents the exudative phase with diffuse alveolar damage, interstitial and alveolar damage followed by a late, fibroproliferative phase with interstitial fibrosis [26]. Among mechanically ventilated ARDS patients, Nakos et al. reported that patients with early ARDS ( $\leq 36 \mathrm{~h}$ from onset) had a superior oxygenation response after prone positioning than those with late ARDS (>36 h from onset) [27]. Similarly, in a prospective study by Coppo et al., the investigators reported an average time between hospital admission to prone positioning of 2.7 days among those who responded to prone positioning, compared to 4.6 days among non-responders in non-intubated patients with COVID-19 [28]. However, their study did not report mortality between the groups. In our study, early APP group received proning within 0.75 days and late APP within 2.5 days of hospital admission. Thus, utilization of early APP during the exudative/inflammatory phase may have led to a better oxygenation response, as well as improvement in work of breathing, which may translate into improved hospital survival.
Second, patients in the early APP group were in the prone position for more total hours than those in the late APP group. On average, the early APP group received $5.07 \mathrm{~h} /$ day of prone positioning as compared to $3 \mathrm{~h} /$ day in the late APP group $(p=0.006)$. Prior studies have demonstrated the mortality benefit of using a longer duration of prone session among intubated patients [3].

Third, a significantly lower number of patients in the early APP group received steroids $(69.6 \%$ vs $87.9 \%$; $p=0.03$ ). The use of steroids is widely reported for clinical management of severe COVID-19 [29]. In one retrospective, observational study steroid use is associated with increased mortality among patients with COVID-19 [30]. In a meta-analysis, the authors reported some beneficial effects of corticosteroids, but note that the overall mortality was higher for those patients that received corticosteroids [31]. A recent open-label randomized controlled (RECOVERY) trial demonstrated a mortality benefit when dexamethasone was used for patients with COVID-19 patients receiving oxygen or IMV [32]. However, another randomized controlled trial evaluating the effectiveness of hydrocortisone in COVID-related ARDS demonstrated no significant reduction in mortality or continued use of respiratory support [33]. In our multivariate analysis, we found that hydrocortisone use, alone or in addition to dexamethasone or prednisone/ methylprednisone, was independently associated with the increased 28-day mortality. This might be explained 
by the likelihood that sicker patients may have received hydrocortisone. Previous studies have demonstrated the increased use of corticosteroids among COVID-19 patients with shock [7]. These study findings suggest further exploration of hydrocortisone use in the treatment of COVID-19 is needed.

This study has several limitations. First, this study is a post hoc analysis of the data collected for a previous randomized clinical trial. Even though post hoc analyses are inherently flawed (due to the lack of randomization, inflated statistical significance, etc.), they do provide exploratory information that could be used to generate hypotheses for future studies [34, 35]. Due to the post hoc nature of this study, we were unable to calculate the sample size to detect the true difference. The sample size in this study is very small which may have impacted the magnitude of an association between the study groups and outcomes [36]. This study included patients from only three hospitals in the USA that may have different clinical management strategies for COVID-19 patients as compared to other clinical settings. Additonally, the original study did not record computed tomography findings to further explore the underlying pathophysiological features associated with increased mortality in the late APP group. Finally, the original study did not collect the incidence of shock or daily disease severity scores that may have prompted the use of steroids such as hydrocortisone among the critically ill patients.

\section{Conclusion}

For patients with acute hypoxemic respiratory failure secondary to COVID-19 and require HFNC therapy, early awake prone positioning ( $<24 \mathrm{~h}$ of HFNC use) is associated with lower 28-day mortality.

\section{Abbreviations}

APP: Awake prone positioning; ARDS: Acute respiratory distress syndrome; AHRF: Acute hypoxemic respiratory failure; CDC: Centers for Disease Control and Prevention; Cl: Confidence interval; COVID-19: Coronavirus disease; ECMO: Extracorporeal membrane oxygenation; HFNC: High-flow nasal cannula; IMV: Invasive mechanical ventilation; OR: Odds ratio; $\mathrm{SpO}_{2}$ : Saturation of pulse oximetry; $\mathrm{FiO}_{2}$ : Fraction of inspired oxygen; ICU: Intensive care unit; NIV: Noninvasive ventilation.

\section{Acknowledgements \\ We thank Jacob Burd, MS, RRT; Kathleen Posa-Kearney, RN; Mark Greenwood, RRT-ACCS; and Scott Heckart, RRT for their help on patient recruitment. We thank the patients and relatives for their participation. We also thank clinical staff members of Respiratory Care department at Rush University Medical Center for providing their support to complete this study.}

\section{Authors' contributions}

$J \mathrm{~L}, \mathrm{RK}$ and DV conceived the idea, JL, RK, SM, AE, JAJ, TW, FC, JBS, IM, MT, IM and LM implemented the study. JL, DV, SM, MT, and IM supervised the study. RK and $J$ conducted data analysis. RK, JL, DV, SM, MT, HH, and IM interpreted the data. RK drafted the manuscript, JL, DV and JBS provided critical revision, all authors reviewed the manuscript for important intellectual content and approved the final manuscript. All authors read and approved the final manuscript.

\section{Funding}

This study was supported by Rice Foundation. The funder had no role in study design, data analysis, the preparation or approval of the manuscript, or the decision to submit the manuscript for publication.

\section{Availability of data and materials}

Any data related question should be directed to the corresponding author.

\section{Declarations}

Ethics approval and consent to participate

The study protocol was approved by the institutional review board (20032604-IRB01).

\section{Consent for publication}

None.

\section{Competing interests}

Dr. Kaur disclose research funding from American Association of Respiratory Care. Dr. Vines discloses research funding from Teleflex Medical, Inc. and Rice Foundation, and speaker fees from Theravance Biopharma. Dr. Scott declares to receive research funding from Teleflex. Dr. Trump discloses consulting fees from Fisher and Paykel. Dr. Li discloses research funding from Fisher \& Paykel Healthcare Ltd, Aerogen Ltd, and Rice Foundation, and speaker fees from American Association for Respiratory Care, Aerogen Ltd, and Fisher \& Paykel Healthcare Ltd. Other authors have no conflict of interest to disclose.

\section{Author details}

${ }^{1}$ Division of Respiratory Care, Department of Cardiopulmonary Sciences, Rush University Medical Center, 600 S Paulina St, Suite 765, Chicago, IL, USA. ${ }^{2}$ Division of Pulmonary, Critical Care, and Sleep Medicine, Rush University Medical Center, Chicago, IL, USA. ${ }^{3}$ Department of Respiratory Care, Unity Point Health-Des Moines, Des Moines, IA, USA. ${ }^{4}$ The lowa Clinic P.C. and Unity Point Health-Des Moines, Des Moines, IA, USA. ${ }^{5}$ Pulmonary and Critical Care Medicine Division, Texas A\&M School of Medicine, Baylor University Medical Center, Dallas, TX, USA. ${ }^{6}$ Nursing, MICU, Rush University Medical Center, Chicago, IL, USA. 'Department of Respiratory and Critical Care Medicine, Beijing Institute of Respiratory Medicine, Beijing Chao-Yang Hospital, Capital Medical University, Beijing, China.

Received: 16 August 2021 Accepted: 4 September 2021

Published online: 17 September 2021

\section{References}

1. World Health Organization. Coronavirus disease 2019 (COVID-19) Situation Report-1. Accessed March 22, 2021

2. Kaur R, Weiss TT, Perez A, et al. Practical strategies to reduce nosocomial transmission to healthcare professionals providing respiratory care to patients with COVID-19. Crit Care. 2020;24(1):571.

3. Guérin C, Reignier J, Richard JC, et al. Prone positioning in severe acute respiratory distress syndrome. N Engl J Med. 2013;368(23):2159-68.

4. Sodhi K, Chanchalani G. Awake proning: current evidence and practical considerations. Indian J Crit Care Med. 2020;24(12):1236-41.

5. Ding L, Wang L, Ma W, He H. Efficacy and safety of early prone positioning combined with HFNC or NIV in moderate to severe ARDS: a multi-center prospective cohort study. Crit Care. 2020;24(1):28.

6. Sud S, Friedrich $\mathrm{JO}$, Taccone $\mathrm{P}$, et al. Prone ventilation reduces mortality in patients with acute respiratory failure and severe hypoxemia: systematic review and meta-analysis. Intensive Care Med. 2010;36(4):585-99.

7. Mathews KS, Soh H, Shaefi S, et al. Prone positioning and survival in mechanically ventilated patients with coronavirus disease 2019-related respiratory failure. Crit Care Med. 2021;49(7):1026-37.

8. Perez-Nieto OR, Escarraman-Martinez D, Guerrero-Gutierrez MA, et al. Awake prone positioning and oxygen therapy in patients with COVID-19: 
The APRONOX study [published online ahead of print, 2021 Jul 15]. Eur Respir J 2021;2100265. doi:https://doi.org/10.1183/13993003.00265-202

9. Taylor SP, Bundy H, Smith WM, Skavroneck S, Taylor B, Kowalkowski MA. Awake-Prone Positioning Strategy for Non-Intubated Hypoxic Patients with COVID-19: A Pilot Trial with Embedded Implementation Evaluation [published online ahead of print, 2020 Dec 23]. Ann Am Thorac Soc 2020;https://doi.org/10.1513/AnnalsATS.202009-1164OC

10. Johnson SA, Horton DJ, Fuller MJ, et al. Patient-Directed Prone Positioning in Awake Patients with COVID-19 Requiring Hospitalization (PAPR) [published online ahead of print, 2021 Feb 17]. Ann Am Thorac Soc 2021; https://doi.org/10.1513/AnnalsATS.202011-1466RL.

11. Rosén J, von Oelreich E, Fors D, et al. Awake prone positioning in patients with hypoxemic respiratory failure due to COVID-19: the PROFLO multicenter randomized clinical trial. Crit Care. 2021;25:209. https://doi.org/10. 1186/s13054-021-03602-9.

12. Kharat A, Dupuis-Lozeron E, Cantero C, et al. Self-proning in COVID-19 patients on low-flow oxygen therapy: a cluster randomised controlled trial. ERJ Open Res 2021;7(1):00692-2020. Published 2021 Mar 8. doi:https://doi.org/10.1183/23120541.00692-2020

13. Jayakumar D, Ramachandran Dnb P, Rabindrarajan Dnb E, Vijayaraghavan Md BKT, Ramakrishnan Ab N, Venkataraman AR. Standard care versus awake prone position in adult nonintubated patients with acute hypoxemic respiratory failure secondary to COVID-19 infection-a multicenter feasibility randomized controlled trial. J Intensive Care Med. 2021;36(8):918-24.

14. Pavlov I, He H, McNicholas B, et al. Awake prone positioning in nonintubated patients with acute hypoxemic respiratory failure due to COVID-19: a systematic review of proportional outcomes comparing observational studies with and without awake prone positioning in the setting of COVID-19 [published online ahead of print, 2021 Jul 7]. Respir Care 2021;respcare.09191. doi:https://doi.org/10.4187/respcare.09191

15. Ehrmann S, Li J, Ibarra-Estrada M, et al. Awake prone positioning for COVID-19 acute hypoxemic respiratory failure: a randomized controlled open-label superiority meta-trial. Lancet Respir Med. 2021:S2213-2600(21):00356-8.

16. Weatherald J, Solverson K, Zuege DJ, Loroff N, Fiest KM, Parhar KKS. Awake prone positioning for COVID-19 hypoxemic respiratory failure: a rapid review. J Crit Care. 2021;61:63-70.

17. Tavernier E, McNicholas B, Pavlov I, et al. Awake prone positioning of hypoxaemic patients with COVID-19: protocol for a randomised controlled open-label superiority meta-trial. BMJ Open 2020;10(11):e041520.

18. Li J, Pavlov I, Laffey JG, et al. Meta-trial of awake prone positioning with nasal high flow therapy: invitation to join a pandemic collaborative research effort. J Crit Care. 2020;60:140-2. https://doi.org/10.1016/j.jcrc. 2020.07.020.

19. Roca O, Caralt B, Messika J, et al. An index combining respiratory rate and oxygenation to predict outcome of nasal high-flow therapy. Am J Respir Crit Care Med. 2019;199(11):1368-76.

20. Gattinoni L, Coppola S, Cressoni M, Busana M, Rossi S, Chiumello D. COVID-19 does not lead to a "typical" acute respiratory distress syndrome. Am J Respir Crit Care Med. 2020;201(10):1299-300.

21. Gattinoni L, Chiumello D, Caironi P, et al. COVID-19 pneumonia: different respiratory treatments for different phenotypes? Intensive Care Med. 2020;46(6):1099-102. https://doi.org/10.1007/s00134-020-06033-2.
22. Marini JJ, Gattinoni L. Management of COVID-19 respiratory distress. JAMA. 2020;323(22):2329-30. https://doi.org/10.1001/jama.2020.6825.

23. Longhini F, Bruni A, Garofalo E, et al. Helmet continuous positive airway pressure and prone positioning: a proposal for an early management of COVID-19 patients. Pulmonology. 2020;26(4):186-91. https://doi.org/10. 1016/j.pulmoe.2020.04.014.

24. Brochard L, Slutsky A, Pesenti A. Mechanical ventilation to minimize progression of lung injury in acute respiratory failure. Am J Respir Crit Care Med. 2017:195(4):438-42. https://doi.org/10.1164/rccm.201605-1081CP.

25. Chatterjee NA, Jensen PN, Harris AW, et al. Admission respiratory status predicts mortality in COVID-19 [published online ahead of print, 2021 May 24]. Influenza Other Respir Viruses 2021. doi:https://doi.org/10.1111/ irv. 12869

26. Matthay MA, Zemans RL, Zimmerman GA, et al. Acute respiratory distress syndrome. Nat Rev Dis Primers. 2019;5(1):18.

27. Nakos G, Tsangaris I, Kostanti E, et al. Effect of the prone position on patients with hydrostatic pulmonary edema compared with patients with acute respiratory distress syndrome and pulmonary fibrosis. Am J Respir Crit Care Med. 2000;161(2 Pt 1):360-8.

28. Coppo A, Bellani G, Winterton D, et al. Feasibility and physiological effects of prone positioning in non-intubated patients with acute respiratory failure due to COVID-19 (PRON-COVID): a prospective cohort study. Lancet Respir Med. 2020;8(8):765-74.

29. Ye Z, Wang Y, Colunga-Lozano LE, et al. Efficacy and safety of corticosteroids in COVID-19 based on evidence for COVID-19, other coronavirus infections, influenza, community-acquired pneumonia and acute respiratory distress syndrome: a systematic review and meta-analysis. CMAJ. 2020;192(27):E756-67. https://doi.org/10.1503/cmaj.200645.

30. Li X, Xu S, Yu M, et al. Risk factors for severity and mortality in adult COVID-19 inpatients in Wuhan. J Allergy Clin Immunol. 2020;146(1):110-8.

31. Cano EJ, Fonseca Fuentes $X$, Corsini Campioli C, et al. Impact of corticosteroids in coronavirus disease 2019 outcomes: systematic review and meta-analysis. Chest. 2021;159(3):1019-40.

32. RECOVERY Collaborative Group, Horby P, Lim WS, et al. Dexamethasone in Hospitalized Patients with Covid-19. N Engl J Med 2021;384(8):693-704.

33. Dequin PF, Heming N, Meziani F, et al. Effect of hydrocortisone on 21-day mortality or respiratory support among critically III patients with COVID19: a randomized clinical trial. JAMA. 2020;324(13):1298-306.

34. Srinivas TR, Ho B, Kang J, Kaplan B. Post hoc analyses: after the facts. Transplantation. 2015;99(1):17-20.

35. Curran-Everett D, Milgrom H. Post-hoc data analysis: benefits and limitations. Curr Opin Allergy Clin Immunol. 2013;13(3):223-4.

36. Hackshaw A. Small studies: strengths and limitations. Eur Respir J. 2008;32(5):1141-3. https://doi.org/10.1183/09031936.00136408.

\section{Publisher's Note}

Springer Nature remains neutral with regard to jurisdictional claims in published maps and institutional affiliations.

Ready to submit your research? Choose BMC and benefit from:

- fast, convenient online submission

- thorough peer review by experienced researchers in your field

- rapid publication on acceptance

- support for research data, including large and complex data types

- gold Open Access which fosters wider collaboration and increased citations

- maximum visibility for your research: over $100 \mathrm{M}$ website views per year

At BMC, research is always in progress.

Learn more biomedcentral.com/submissions 